\title{
Comportamiento y organización. Implementación del sistema de gestión de la calidad $5 S^{\prime} S^{*}$
}

\section{Behavior and Organization. Implementation of Quality Management System 5 S'S}

\author{
Felipe Santoyo Telles ${ }^{* * *}$ \\ Centro de Investigaciones en \\ Comportamiento Alimentario y \\ Nutrición (CICAN), Universidad de \\ Guadalajara \\ Daniel Murguía Pérez \\ Instituto Tecnológico de Cd. \\ Guzmán \\ Antonio López-Espinoza \\ Centro de Investigaciones en \\ Comportamiento Alimentario y \\ Nutrición (CICAN), \\ Centro Universitario del Sur \\ (CUSur) de la Universidad de \\ Guadalajara \\ Eliseo Santoyo Teyes \\ Centro de Bachillerato Tecnológico \\ Industrial y de Servicios 226 \\ Universidad de Guadalajara \\ Centro Universitario del Sur \\ (CUSur) de la Universidad de \\ Guadalajara, México \\ Recibido: 27 de enero de 2013 \\ Revisado: 7 de marzo de 2013 \\ Aceptado: 17 de mayo de 2013
}

\section{Resumen}

Con la finalidad de mejorar los servicios que ofrece, las condiciones físicas de instalaciones y equipo, y con ello generar una cultura de calidad en el trabajo, el Departamento de Recursos Materiales y Servicios del Instituto Tecnológico de Ciudad Guzmán (ITCG) es detectado como un área de oportunidad. Como propuesta de solución para mejorar esta área se implementó el sistema de administración de calidad 5S, una técnica de trabajo que crea un cambio en el comportamiento de los trabajadores, basada en cinco aspectos: clasificación, orden y limpieza, reforzado con estandarización y disciplina. En el presente trabajo se demuestra que el aprendizaje de la metodología $5 \mathrm{~S}$ modifica el comportamiento, el ambiente de trabajo de un departamento y/o organización, propiciando el trabajo en equipo, para mejorar la comunicación, incrementar la motivación del personal y facilitar el cumplimiento de los objetivos establecidos. Los resultados demostraron que se logró establecer orden y limpieza; reducir el tiempo de búsqueda por herramienta en un $80 \%$ y de insumos en un $66,6 \%$, y una ganancia de $20 \mathrm{~m}^{2}$ en espacios ganados.

Palabras clave: conducta 5S, mejoramiento continuo, Kaizen, gestión de la calidad.

\footnotetext{
Artículo de investigación. Centro de Investigaciones en Comportamiento Alimentario y nutrición (CICAN), Centro Universitario del Sur (CUSur) de la Universidad de Guadalajara

** Correspondencia: Felipe Santoyo Telles. Centro Universitario del Sur - Universidad de Guadalajara. Av. Enrique Arreola Silva No. 883, Edificio X-3, Colonia Centro C.P. 49000. Ciudad Guzmán, municipio de Zapotlán el Grande, Jalisco. México. Correo electrónico: felipes@cusur.udg.mx
} 


\begin{abstract}
With the objective of improve services to public, equipment and facilities conditions, Department of material resources and services of technological Institute of Ciudad Guzmán (ITCG) is identified as an area of opportunity to generate a culture of quality in the work.. As a proposed solution to improve this area, a system of quality management was implemented (5s's), the working technique that creates a change in habits of workers, based on Classification, Order and Cleanliness, Standardization and Discipline reinforce. The present work shows that learning of the methodology 5S's, modifies; behavior, working environment of a department or organization, promoting teamwork, improving communication, increasing staff motivation and achievement of specific objectives. Results showed that it was to establish order and cleanliness; reduce search time by tool in $80 \%$, on inputs a $66.6 \%$ and a gain of $20 \mathrm{~m}^{2}$ in cattle spaces.
\end{abstract}

Keywords: behavior, 5S, continuous improvement, Kaizen, quality management.

\section{Introducción}

Al momento de planificar la mejora continua en las organizaciones frecuentemente nos vemos atraídos por soluciones complicadas; hablar de organizar, ordenar y limpiar puede ser considerado como algo trivial o demasiado simple, ya que son conceptos que se asocian con el ámbito doméstico y nunca el empresarial. Sin embargo, estos tres conceptos se hallan íntimamente ligados con la gestión. Son el primer paso que debe dar una empresa en su proceso para modificar el comportamiento de los empleados. Esta acción es una premisa básica e imprescindible para aumentar la productividad y conseguir un entorno seguro y agradable (Rovira, 2011).

Organizar, ordenar y limpiar son actividades que no están en contra de los procesos de mejora continua, ya que son fundamentales para poder identificar los problemas y/o procesos que se quieran mejorar en una organización cualquiera que sea su objetivo; si no se cuenta con un lugar de trabajo limpio y bien ordenado, este se convierte en una zona de peligro y, en consecuencia, es casi imposible detectar dónde están los problemas.

Sin embargo, un elemento indispensable en la implementación de cambios conductuales dirigidos a la detección y corrección de errores en un sistema se basa en el aprendizaje organizacional.
Con este tipo de aprendizaje es posible modificar la imagen de una organización a partir de la comprensión de los fenómenos organizacionales que son la base para reestructurar las actividades cotidianas, con el objetivo de que tanto resultados como expectativas coincidan. Este tipo de aprendizaje cambia el comportamiento de los integrantes de la organización. En este sentido, establecer el aprendizaje de métodos o sistemas específicos, dirigidos a modificar la conducta organizacional de un determinado sistema, son la primera etapa de todo un proceso de cambio (Rey, 2005). Así, incorporar el sistema de las $5 S$ es un método práctico, sencillo y de fácil aprendizaje.

Las 5 S es una filosofía de trabajo que permite desarrollar un comportamiento sistemático para mantener continuamente la clasificación, el orden y la limpieza, lo que permite de forma inmediata una mayor productividad, mejorar la seguridad, el clima laboral, la motivación del personal, la calidad, la eficiencia y, en consecuencia, la competitividad de la organización (Barcia, 2006; Ho, 1997). De acuerdo con Ruiz (2003), la metodología está dirigida a mejorar el entorno de los procesos, sin modificar necesariamente su esencia; Ugalde (1988), por su parte, citado en Granados (2001), asegura que $\sin$ las 55 , todas las otras metodologías, herramientas de calidad, sistemas y/o mecanismos para obtener mayor productividad o para mejorar el ambiente de trabajo son tiempo perdido. 
Las $5 \mathrm{~S}$ son útiles no solo para mejorar el entorno físico, sino, también, para mejorar el proceso del pensamiento (Ho, 1997; Sui-Pheng y Khoo, 2001). En este contexto, la gestión de la organización y del orden, la gestión visual, el hábito y la autodisciplina propuestos por la metodología de las $5 \mathrm{~S}$ pueden ser considerados moduladores de las esferas de los procesos perceptivos, procesos emocionales, procesos cognitivos y procesos ejecutivos (López, 2004).

El presente trabajo tiene como objetivo implementar el sistema de administración de la calidad 5S, para modificar el comportamiento y lograr un mejoramiento continuo en el personal y las condiciones del Departamento de Recursos Materiales y Servicios del Instituto Tecnológico de Ciudad Guzmán (ITCG). Esta metodología fue elaborada por Hiroyoki Hirano, y se denomina 5S debido a las iniciales de las palabras japonesas seiri, seiton, seiso, seiketsu y shitsuk, que significan clasificación, orden, limpieza, estandarización y disciplina (Soto, 2007; Feld, 2001).

El sistema de las $5 S$ se define como el cambio de conducta para organizar el lugar de trabajo, conservarlo limpio, con condiciones de trabajo estandarizadas, reforzado con una actitud disciplinada. Las $5 \mathrm{~S}$ son la herramienta clave para lograr la organización del área de trabajo (Euskalit, 2008), y cada concepto tiene una función particular. Seiri: selección o clasificación, distinguir lo que es necesario de lo que no lo es. Seiton: orden u organización, un lugar para cada cosa, y cada cosa en su lugar. Seiso: limpieza, establecer métodos para mantener limpio el lugar de trabajo. Seiketsu: limpieza estandarizada, es la condición o el estado que existe cuando se mantienen apropiadamente las tres primeras "S" (selección, organización y limpieza). Shitsuke: disciplina, establecer mecanismos para hacerlo un hábito. Cumplir con el compromiso que se adquiere al aceptar un trabajo.

Un área con características específicas para la aplicación del sistema $5 \mathrm{~S}$ es el Departamento de Recursos Materiales y Servicios Generales del Instituto Tecnológico de Ciudad Guzmán. Este Tecnológico se ubica a 3,5 km de Ciudad Guzmán,
Jalisco, México, sobre la carretera al FresnitoCiudad Guzmán (región sur del estado de Jalisco). Actualmente cuenta con ocho ingenierías, tres licenciaturas y dos maestrías, la población escolar para el periodo escolar enero-junio de 2011 es de aproximadamente 4.000 estudiantes (Gastélum, 2011). Para el periodo enero-junio de 2011, el ITCG contaba con 284 empleados, de los cuales 161 correspondían a personal docente; 91 , a administradores, y 21 eran técnicos docentes.

El Departamento de Recursos Materiales y Servicios Generales está constituido por 40 personas. De los 40 trabajadores, 10 son mujeres y 30 son hombres. Respecto a las edades, el 30\% está en los rangos de 31 a 40, seguido por el $25 \%$ que están en los rangos de 61-70. Es importante señalar que, a pesar de que se cuenta con una mayoría de trabajadores jóvenes (12 trabajadores en el rango de 31 a 40 años), también existen 10 trabajadores en el rango de 61 a 70 años. Respecto al nivel escolar, el $45 \%$ tiene la primaria terminada y solo el 7,5\% del personal informa haber terminado una carrera profesional.

En el Departamento de Recursos Materiales y Servicios del Instituto Tecnológico de Ciudad Guzmán, al momento de la intervención no se tenía un control adecuado de los productos que se utilizan tanto en el almacén como en las áreas de trabajo (mantenimiento, limpieza, vigilancia y operadores). Era visible que en las oficinas, almacén y áreas de trabajo no se cuenta con un comportamiento de limpieza estandarizada. Se detectó que las personas que laboran en este departamento no conocían la ubicación correcta de las herramientas de trabajo, lo cual ocasionaba una pérdida de tiempo de 15 a 20 minutos por búsqueda. En los almacenes - sobre el piso- se encontraban herramientas y materiales que obstruían el libre tránsito, lo que ocasionaba una demora de 5 a 7 minutos en cada trabajo realizado; así mismo, por la demora de la actividad cotidiana se generaba un ambiente de tensión y disgusto en el trabajador.

En el área de oficinas se localizaban cajas con material para impresión que obstaculizaban el movimiento propio de las áreas de trabajo. Fue posible 
detectar que una de las necesidades prioritarias de las personas que laboran en el departamento es contar con lugares adecuados para practicar con calidad cada una de las actividades de su vida, entre las cuales está el trabajo. Cuando el entorno de trabajo está desorganizado y sin limpieza, se pierde la eficiencia y se reduce el entusiasmo por el trabajo (MP, 2013).

La hipótesis del presente trabajo parte de la siguiente premisa: con la implementación del modelo $5 \mathrm{~S}$ se modificará el comportamiento para lograr volver eficiente el uso de espacio útil, reducir los tiempos de ubicación y acceso a herramientas de trabajo y materiales, facilitando así el control visual de todos ellos. La limpieza y aseo se harán con mayor facilidad y seguridad, lo que dará una mejor presentación al departamento, incrementará la vida útil tanto de herramientas como de equipo al evitar su deterioro por contaminación y, por consiguiente, mejorará el ambiente de trabajo.

\section{Estrategias de implementación}

Para poder alcanzar los objetivos propuestos en el presente trabajo y además poder comprobar la hipótesis establecida se seguirá la metodología de implementación del sistema de administración de la calidad 5S. Con el objetivo de conocer la situación del Departamento de Recursos Materiales y Servicios se aplicó la primera estrategia, el elemento sorpresa, el cual consistió en esto: durante un día normal de trabajo se tomaron fotografías de las diferentes áreas del departamento, para tener evidencia que revelara el estatus que guardan el orden y la limpieza.

Como segunda estrategia se citó a los 40 integrantes del Departamento en la sala de capacitación del Instituto, en dicha reunión se proyectó la galería fotográfica producto de la aplicación de la primera estrategia, con el objetivo de establecer el primer paso del aprendizaje organizacional, al señalar la importancia que tiene la aplicación de la metodología del sistema de administración de la calidad 5 S.

En la tercera estrategia se aplicó un cuestionario de auditoría (Montero, 2000), el cual evaluó, por medio de un rango de valor 0 como mínimo y 100 como valor máximo, el grado de aplicación de las $5 \mathrm{~S}$ en la organización. El instrumento fue aplicado al $100 \%$ de la población de estudio, que consistió en las 40 personas del Departamento de Recursos Materiales del ITCG.

Finalmente, en la cuarta estrategia se elaboró un instrumento para medir el impacto de la metodología basado en la productividad. Dicho instrumento basa su medición en dos grandes rubros:

- Ahorro de tiempos muertos (ocasionados por la búsqueda de herramientas e insumos): se registraron las mediciones de tiempos muertos en los primeros tres días de arranque del proyecto y se contrastaron con las medidas obtenidas al finalizar el proyecto.

- Ganancia de espacios (obtenidos por la disminución de consumibles e inventarios): se midieron los espacios que se generaron por la remoción de elementos que se reacomodaron.

A continuación se presentan los resultados obtenidos tanto en el diagnóstico como en la aplicación de la metodología $5 \mathrm{~S}$.

\section{Resultados}

\section{Exposición informativa}

Esta exposición (segunda estrategia) tuvo como objetivo la modificación del comportamiento del personal que trabaja en todas las áreas del Departamento, a partir del adecuado conocimiento (aprendizaje organizacional) del método de trabajo 5S; para ello, se hizo una sesión de trabajo de 30 horas, en la cual se abordaron los siguientes puntos:

- ¿Qué son las 5S?

- ¿Para qué sirven?

- ¿En qué nos benefician?

- Establecer puntos de mejora en el departamento.

En todo momento se observó un liderazgo participativo por parte del jefe del departamento y de 
sus encargados de oficina. Es importante señalar que los factores influyentes para una implementación exitosa de métodos productivos radican en que el liderazgo y la responsabilidad sean elementos compartidos entre la alta administración y los equipos de trabajo (Espinoza y Hejduk, 2010).

En esta primera etapa todo el personal se comprometió con la implementación del sistema 5S. Así, se establecieron las siguientes metas:

- El tiempo de aplicación de la primera S, selección (seiri), no mayor a 15 días hábiles en todas las áreas.

- El tiempo de aplicación de la segunda S, orden (seiton), no mayor a 15 días hábiles en todas las áreas.

- El tiempo de aplicación de la tercera S, limpieza (seiso), no mayor a 10 días hábiles en todas las áreas.

- El tiempo de aplicación de la cuarta S, limpieza estandarizada (seiketsu), permanecería constante en todas las áreas del departamento.

- El tiempo de aplicación de la quinta S, disciplina (shitsuke), permanecería constante en todas las áreas del departamento.

Entre otras interrogantes que se aclararon, también se hizo una evaluación mediante fotos (primera estrategia) para conocer la situación real del departamento antes de implementar el sistema de las 5S. En la tabla 1 podemos ver los resultados de la inspección inicial.

Tabla 1.

Resultados de la inspección inicial 5S

\begin{tabular}{lccc}
\hline Conceptos & Calificación & Máximo & $\%$ \\
\hline Clasificación & 8 & 20 & 40 \\
Orden & 2 & 20 & 10 \\
Limpieza & 1 & 20 & 5 \\
Estandarización & 3 & 20 & 15 \\
Disciplina & 6 & 20 & 30 \\
Total & 20 & 100 & 20 \\
\hline
\end{tabular}

Al aplicar el instrumento de auditoría 5S (Montero, 2000) fue posible observar que el nivel de esta estrategia en el departamento resultó bajo (calificación de 20 sobre 100 puntos); la limpieza es el apartado con menos puntaje, la principal razón es la falta de estandarización de esta, ya que se practica eventualmente de manera superficial y no profunda.

\section{Condiciones al momento de la intervención}

El departamento cuenta con un total de seis áreas de trabajo, área de oficinas, casilleros, herrería y taller de soldadura, comedor, área de mecánica y de cubículos; por cuestiones de espacio solo se detallarán dos de las principales áreas problemas, así mismo, se hará mención de los problemas en común que se encontraron para todas las áreas.

\section{Área de oficinas del edificio}

En la figura 1 se muestra el área de oficinas en la cual se desarrolla el proceso administrativo del Departamento de Recursos Materiales y Servicios.

Figura 1. Área de oficinas
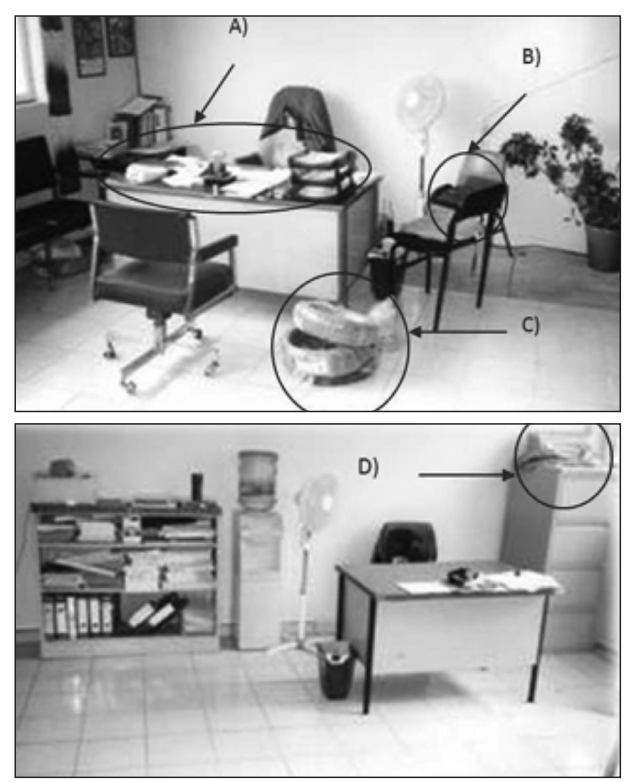
En la figura 1 es visible la desorganización, objetos de oficina que están en lugares inadecuados, escritorios con documentación por archivar que hacen caso a la falsa creencia de que "entre más papeles o cosas tengamos en áreas de trabajo" parecerá que somos más trabajadores y productivos.

a. El escritorio se encuentra en desorden, lo cual hace una tarea difícil encontrar documentos a simple vista.

b. El fax no cuenta con un lugar adecuado; está actualmente sobre una silla con el riesgo de caer y quedar inhabilitado.

c. Las mangueras están en el lugar incorrecto, esto genera una condición insegura para el personal que trabaja en esta área. Este tipo de material debe tener un lugar definido en el almacén.

d. La impresora se encuentra obsoleta, por lo cual solo está ocupando espacio que puede ser útil para otros elementos de oficina.

\section{Área de casilleros}

Esta área cuenta con 22 casilleros, los cuales fueron distribuidos en modo personalizado, utilizados para guardar pertenencias los trabajadores. El centro del área cuenta con un pasillo, el cual debe de estar limpio y accesible a cada casillero.

En la figura 2, correspondiente al área de casilleros, se observan:

Figura 2. Área de casilleros

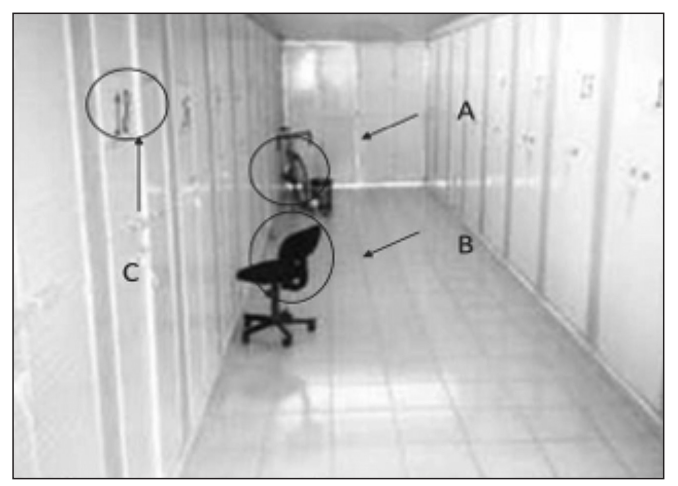

a. Botes de pintura y bolsas de papel obstruyendo la entrada a uno de los casilleros.

b. La silla se encuentra en un área inadecuada, que da una mala imagen del lugar.

c. Los nombres correspondientes de cada trabajador escritos en los casilleros están en hojas blancas, lo cual demerita la imagen del lugar.

\section{Área de herrería y taller de soldadura}

Esta área se utiliza para ejecutar los trabajos de herrería y mantenimiento de butacas, escritorios, sillas, puertas, mesas, registros, etc.

En la figura 3, correspondiente al área de herrería y taller de soldadura, se observa:

Figura 3. Área de taller

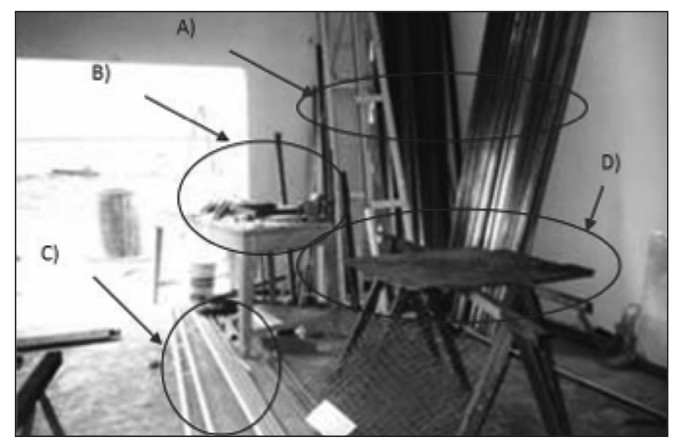

a. Materia prima, tubos de PTR, solera, etc. Estos se encuentran en posición vertical, lo cual, debido a la longitud y peso del material, genera un riesgo para el personal.

b. En el banco de trabajo se puede apreciar material sobrante de trabajos concluidos y herramientas de trabajo que no se ubican en el lugar designado.

c. El material se visualiza en el piso, obstruyendo el libre tránsito en el área de trabajo, lo cual ocasiona una situación de riesgo.

d. Podemos observar que los trabajadores improvisan mesas de trabajo sobre herramientas utilizando un material inadecuado. En dicho banco se muestra una clara inestabilidad para la ejecución de trabajos. 
Figura 4. Área de taller

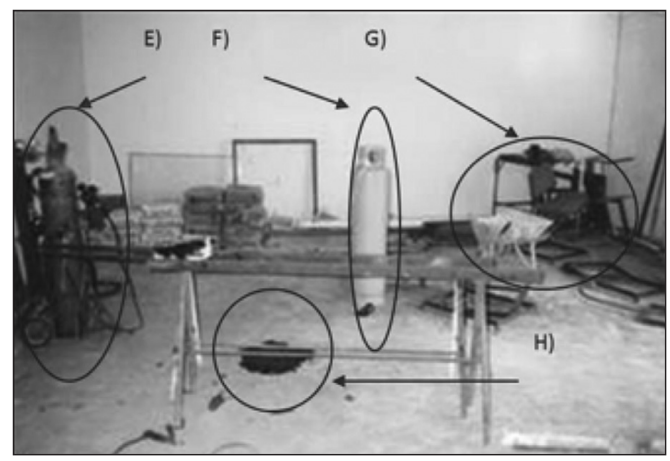

a. El equipo de soldadura autógena tiene dos mangueras y en sus extremos, la herramienta para soldadura o corte; es visible que están sobre el piso, lo cual genera un deterioro prematuro del equipo (figura 4).

b. El tanque de gas se encuentra entre el equipo de soldadura eléctrica y el equipo de soldadura autógena, sin soporte o base (figura 4).

c. Las bancas y butacas que están en espera de reparación se almacenan de una forma inadecuada dentro del área, lo que ocasiona una reducción del espacio de trabajo (figura 4).

d. Se observa una mancha de aceite en el piso, que representa un riesgo para el trabajador y un mal aspecto para el área (figura 4).

En conclusión, estas áreas cuentan con una mala organización, tanto de las áreas como de herramientas y materiales que se utilizan en el trabajo; el piso se encuentra en un estado de alta suciedad, en el cual podemos encontrar hojas de papel, cartón, aceite, rebabas, etc. No se cuenta con un programa de limpieza estandarizado y sí con un alto grado de riesgo para los trabajadores.

Por su parte, en el área del comedor, las sillas y mesas que se utilizan no son adecuadas y dan mal aspecto al lugar. En el área de mecánica, la fosa destinada a dar mantenimiento a equipos móviles se encuentra llena de basura; se hallaron diversos materiales, desde cartón, plástico, butacas, envases de refresco vacíos, ladrillos y materiales de construcción y otros desechos que impiden que se realicen las labores para la cual fue diseñada, hasta un par de podadoras inservibles que reducen el área de mantenimiento.

El área de cubículos está asignada al personal de mantenimiento, en ellos cada trabajador tiene sus herramientas de trabajo. En el área permanecen materiales amontonados y desordenados, cubetas de pintura en el piso que obstruyen el paso y pueden causar un accidente, la estantería contiene materiales desordenados y que ya no tienen ningún uso práctico, se encontraron herramientas y materiales de las que no se puede identificar si pertenecen al área.

\section{Postaplicación del sistema de calidad 5S}

Al eliminar materiales innecesarios se generaron nuevos espacios útiles en oficinas, pasillos, casilleros y cubículos, en áreas donde regularmente se encontraban objetos innecesarios y basura. Al término de este proyecto las áreas se encuentran limpias, gracias a un plan de trabajo de limpieza estandarizada (figura 5).

Figura 5. Área de casilleros

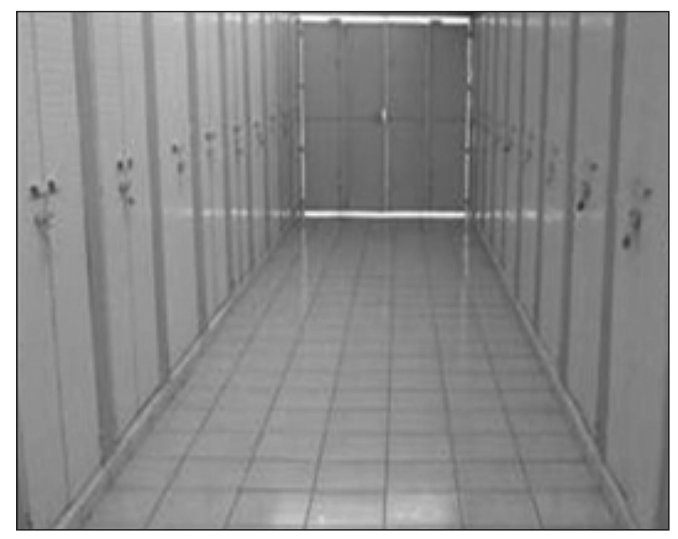

En la oficina se logró un mejoramiento considerable, tanto en limpieza como en orden; los escritorios dan un aspecto higiénico, en el que sobresale la distribución de documentos; los equipos de oficina, como radios y fax, ya cuentan con un área específica, como se ilustra en la figura 6. 
Figura 6. Área de oficinas
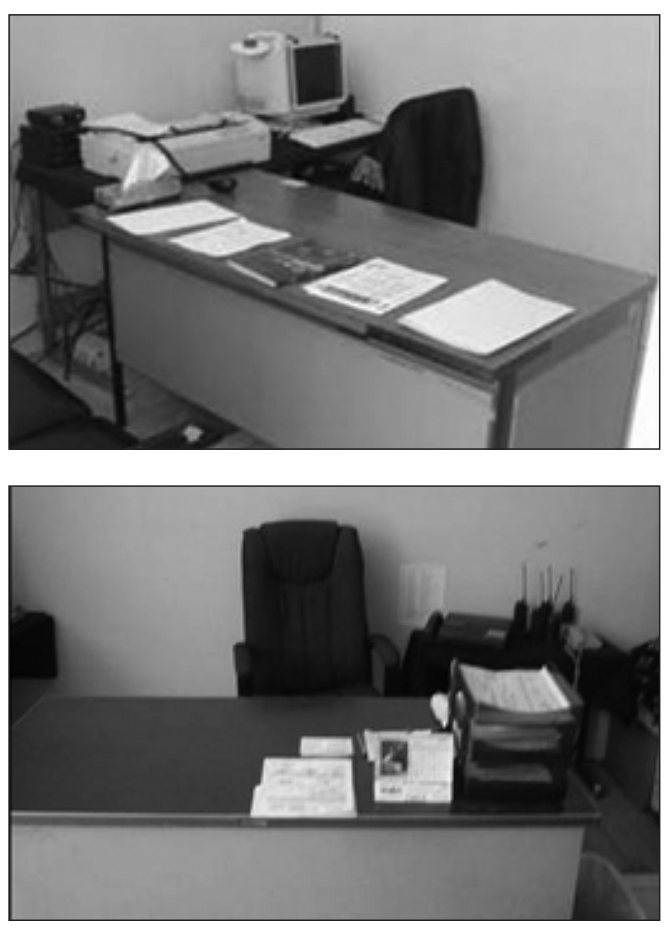

En el área de oficina, durante el transcurso de la jornada los trabajadores mantienen un orden en cuanto a la documentación que se utiliza, como se muestra en la figura 6. Al terminar las jornadas de trabajo es notorio el orden y la limpieza con que se dejan las áreas de trabajo, como se muestra en la figura 7.

Figura 7. Área de oficinas

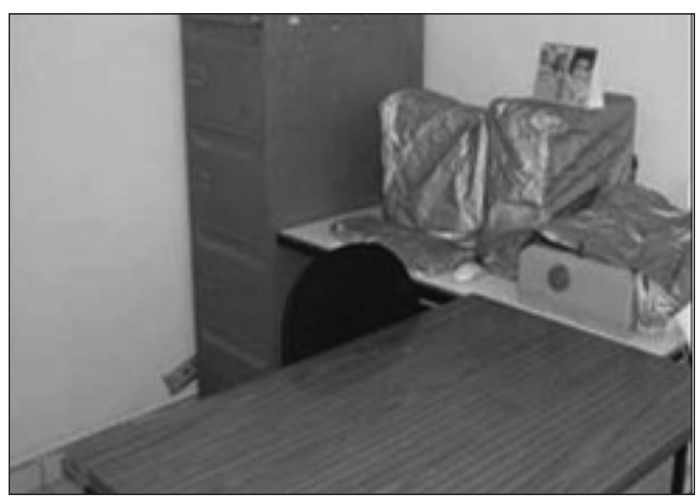

\section{Indicadores de productividad y cambio de comportamiento}

La tabla 2 nos muestra la comparación de los indicadores de productividad antes y después de la implementación del sistema 5 S.

Tabla 2.

\begin{tabular}{lccc}
\hline $\begin{array}{l}\text { Indicadores de } \\
\text { productividad } \\
\text { Indicador }\end{array}$ & $\begin{array}{c}\text { Antes de } \\
\text { las 5S }\end{array}$ & $\begin{array}{c}\text { Después } \\
\text { de las 5S }\end{array}$ & $\begin{array}{c}\text { Porcentaje } \\
\text { de mejora }\end{array}$ \\
\hline $\begin{array}{l}\text { Tiempo de } \\
\text { búsqueda de } \\
\text { herramientas }\end{array}$ & 10 & 5 & $50 \%$ \\
$\begin{array}{l}\text { Tiempo de } \\
\text { búsqueda de } \\
\text { utilerías }\end{array}$ & 6 & 2 & $33,33 \%$ \\
$\begin{array}{l}\text { Espacio ganado } \\
\text { (despeje de } \\
\text { zonas) }\end{array}$ & $0 \mathrm{~m}^{2}$ & $20 \mathrm{~m}^{2}$ & \\
\hline
\end{tabular}

\section{Resultados de la inspección al final de la estrategia de las $5 \mathrm{~S}$}

La tabla 3 nos muestra los resultados obtenidos en la inspección al final de la implementación del sistema $5 \mathrm{~S}$.

Tabla 3.

Resultados de la inspección final

\begin{tabular}{lccc}
\hline Pilar & Calificación & Máximo & $\%$ \\
\hline Clasificación & 15 & 20 & 75 \\
Orden & 20 & 20 & 100 \\
Limpieza & 20 & 20 & 100 \\
Estandarización & 12 & 20 & 60 \\
Disciplina & 15 & 20 & 75 \\
Total & $\mathbf{8 2}$ & $\mathbf{1 0 0}$ & $\mathbf{8 2}$ \\
\hline
\end{tabular}

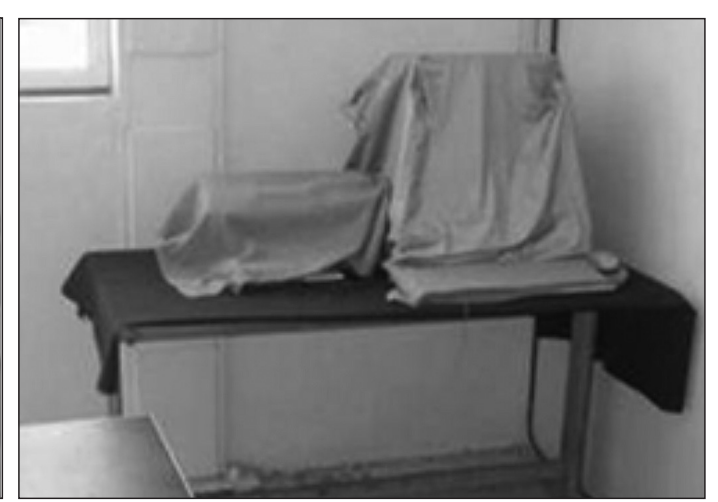


Al aplicar inicialmente el cuestionario de auditoría $5 S$ (Montero, 2000) se pudo observar que dicha aplicación en el área era demasiado baja, con una calificación de 20 sobre 100 puntos; posterior a la aplicación de la estrategia el resultado se incrementó a 82 sobre 100. Como resultado directo de la aplicación de la metodología de las 55 podemos observar que los pilares de limpieza y orden fueron los más sobresalientes, con un puntaje de 20 sobre 20. El tiempo que pasó entre la aplicación por primera vez del cuestionario de auditoría 5S (Montero, 2000) y la segunda aplicación se da en un periodo no mayor a 45 días.

\section{Discusión}

Los resultados obtenidos en el presente estudio mostraron que con la aplicación de la metodología 5S: a. se redujo el tiempo de búsqueda por herramienta en un $80 \%$; b. se redujeron los tiempos de búsqueda de insumos en un 66,6\%; se incrementó en $20 \mathrm{~m}^{2}$ los espacios ganados; y c. la evaluación del nivel del conocimiento de las 55 nos informó un incremento del 60\%. Los resultados demuestran que la implementación de la estrategia de las $5 \mathrm{~S}$ modificó el comportamiento del personal del Departamento de Recursos Materiales y Servicios del Instituto Tecnológico de Ciudad Guzmán. Es necesario considerar que un elemento de importancia es el proceso de educación-aprendizaje para la obtención de resultados.

Para una adecuada implementación de la estrategia de las $5 \mathrm{~S}$ es de suma importancia practicar una adecuada modificación del comportamiento, por lo que se requiere programar y dedicar el tiempo y recursos necesarios para que el personal comprenda, aprenda y aplique de manera adecuada los pasos de la estrategia. La evidencia experimental ha demostrado que esta es la etapa crítica para lograr la mejora continua en cualquier tipo de empresa o sistema (Gudiño y Cedillo-Campo, 2011; Juarez, 2009; Torres, 2011). Consideramos que nuestro trabajo estableció una adecuada estrategia para informar y enseñar al personal involucrado en el Departamento, razón por la cual obtuvimos resultados muy favorables.
Juarez (2009) señaló que un elemento indispensable para que una organización subsista es su grado de competitividad. Para lograr tal elemento es necesario que las organizaciones desarrollen una dinámica de mejoramiento continuo, y esto no es posible sin la colaboración de cada uno de los integrantes de la organización. En este sentido, en nuestro trabajo se involucró tanto a personal del ámbito operativo como del ámbito gerencial, esto permitió que la implementación de la estrategia de las $5 S$ tuviera éxito.

Es necesario considerar que existen factores que pueden intervenir y afectar directamente la implementación de la estrategia de las 5S. Torres (2011) señaló que existen al menos tres factores que son determinantes para la implementación de las 5S; el primero de ellos se refiere al compromiso que los diferentes niveles jerárquicos de la organización o sistema establezcan con la implementación de la estrategia de las 5S. El posible éxito o fracaso dependerá, en gran medida, de que estos niveles jerárquicos integren en la visión de desarrollo de la organización la incorporación de dicha estrategia. Un segundo elemento se refiere a la necesidad de integrar en un solo nivel jerárquico la visión y toma de decisiones relacionados con la implementación de la estrategia y la toma de decisiones que esta involucra.

Finalmente, el factor más relevante es la modificación del comportamiento de quienes integran la organización en la que se aplica la mejora continua; para ello es necesario crear un ambiente de disposición al cambio o de lo contrario la estrategia fracasará. En este sentido, es importante mencionar que el éxito o el fracaso del sistema de calidad 5S depende de la constancia y el compromiso de sus aplicadores, ya que si no se esfuerzan por cumplir con las normas y estandarizaciones no podrán ver resultados concretos, como lo señala Liker: “ [...] una dirección comprometida, una formación adecuada y una cultura que haga que la mejora sostenida sea el comportamiento habitual desde el taller hasta la dirección” (2004, p. 74).

Es posible afirmar que los resultados obtenidos en el presente trabajo demuestran la efectividad de la implementación de la estrategia de las 5S. 
Es necesario señalar que para obtener el éxito en el presente trabajo se conjuntaron voluntades tanto de directivos como del personal operático; sin embargo, lo que fue determinante es la voluntad de cambio basada en una adecuada estrategia para transmitir los objetivos y alcances de la implementación de la estrategia de las 5S. Sera necesario aplicar una evaluación futura de los alcances que este trabajo dejó en el Departamento de Recursos Materiales y Servicios del Instituto Tecnológico de Ciudad Guzmán para reforzar lo implementado o rectificar en caso necesario.

\section{Referencias}

Barcia, F., Hidalgo, D. (2006). Implementación de una metodología con la técnica $5 S$ para mejorar el área de matricería de una empresa extrusora de aluminio. Revista Tecnológica ESPOL, 18(1), 69-75.

Espinoza, M., \& Hejduk, I. (2010). Modelo de administración de la mejora continua para pequeñas y medianas empresas mexicanas. Ide@s-CONCYTEG, 5(65), 1307-1334. EUSKALIT. (2008). Metodología de las $5 \mathrm{~S}$ mayor productividad mejor lugar de trabajo. Recuperado de http://www.euskalit.net/pdf/ folleto2.pdf

Feld, W. M. (2001). Lean manufacturing: tools, techniques and how to use them. Recuperado de http://es.scribd.com/doc/59016472/LeanManufacturing-Tools-Techniques-and-How-toUse-Them

Gastélum, G. (2011). Estadístico. Datos estadísticos del Instituto Tecnológico de Ciudad Guzmán. Recuperado de http://www.itcdguzman.edu.mx/

Granados, S. M. (2001). Propuesta de la estrategia 55 como medio para implementar la cultura de mejoramiento continuo en el departamento de control de materiales de la empresa VTECH de México Reynosa, S. A. de C. V. (Tesis no publicada, Maestría en Gestión de la Calidad, México, Universidad Autónoma de Tamaulipas).
Gudiño, G., \& Cedillo-Campo, G. (2011). Diseño de un sistema de producción específico para operaciones en mercados emergentes. Interciencia, 36, 456-462.

Ho, S. (1997). Workplace Learning: the 5-S Way. Journal of Workplace Learning. 9(6), 85-91.

Juarez, G. K. V. (2009). Propuesta para implementar metodología 5S's en el departamento de cobros de la subdelegación Veracruz norte IMSS. Tesis inédita.

Liker, J. (2004). The Toyota way. New York: Free Press.

López Fresno, P., \& Fernández-González, F. (2004). Environmental Management Through 5-S. Proceedings of the 7 th International Conference on ISO 9000 and Total Quality Management. Banghkok.

Mantenimiento planificado. (s. f.). 5 S's en profundidad. Recuperado de http://www.mantenimientoplanificado.com/Articulos\%20gestión\%20mantenimiento_archivos/Manual\%20 Por\%20que\%20se\%20llama\%20estrategia\%20 de\%20las\%20cinco\%205s.pdf

Montero, H. (2000). Implementación de las $5 \mathrm{~S}$. Herramienta japonesa para el Orden y Limpieza. Biblioteca Virtual de la Sociedad Latinoamericana para la Calidad. Recuperado de http://www.calidad.org

Rey, F. (2005). Las 5S. Orden y limpieza en el puesto de trabajo. Madrid: FC Editorial.

Rovira, C. (2011). Implementación de sistemas de gestión. Recuperado de http:// www.op-group.net/

Ruiz, J. (2003). Breve análisis de la evolución, innovación y mejores prácticas de los procesos industriales, Boletín IIE. Aplicaciones tecnológicas. Recuperado de http://www.iie.org. $\mathrm{mx} /$ boletin032003/aplica.pdfl_

Soto, R. (2007). El proceso de las 5 S's en acción: La metodología Japonesa para mejorar la ca- 
Comportamiento y organización. Implementación del sistema de gestión de la calidad 5S’S

lidad y la productividad de cualquier tipo de empresa. Revista Gestión y Estrategia, 1(31), 91-94.

Sui-Pheng, L., \& Khoo, S. D. (2001). Team performance management: enhancement through
Japanese 5-S Principles. Team Performance Management, 7(7/8), 105-111.

Torres, J. (2011). Mejora Continua: Implementación de las $5 \mathrm{~S}$ en un Sistema de Salud. Interciencia, 1, 33-37. 